\title{
Retention Behaviour in Micellar Liquid Chromatography
}

\author{
Maria Rambla-Alegre \\ Àrea de Química Analítica, QFA, Universitat Jaume I, 12071 Castelló, Spain \\ Correspondence should be addressed to Maria Rambla-Alegre,mrambla@guest.uji.es \\ Received 30 November 2011; Accepted 11 January 2012 \\ Academic Editor: Samuel Carda-Broch
}

Copyright (C) 2012 Maria Rambla-Alegre. This is an open access article distributed under the Creative Commons Attribution License, which permits unrestricted use, distribution, and reproduction in any medium, provided the original work is properly cited.

Retention in micellar liquid chromatography is highly reproducible and can be modelled using empirical or mechanistic models with great accuracy to predict the retention changes when the mobile phase composition varies (surfactant and organic solvent concentrations), thus facilitating the optimisation of separation conditions. In addition, the different equilibria inside the column among the solute, the mobile phase, and the modified stationary phase by monomers of surfactant have been exhaustively studied. In a sequential strategy, the retention of the solutes is not known a priori, and each set of mobile phases is designed by taking into account the retention observed with previous eluents. By contrast, in an interpretative strategy, the experiments are designed before the optimization process and used to fit a model that will allow the prediction of the retention of each solute. This strategy is more efficient and reliable. The sequential strategy will be inadequate when several local and/or secondary maxima exist, as frequently occurs in chromatography, and may not give the best maximum, that is to say, the optimum. More often than not, the complexity of the mixtures of compounds studied and the relevant modification of their chromatographic behaviour when changing the mobile phase composition requires the use of computer-assisted simulations in MLC to follow the modifications in the chromatograms in detail. These simulations can be done with sound reliability thanks to the use of chemometrics tools.

\section{Introduction}

Most reported procedures for the determination of compounds in MLC make use of micellar mobile phases containing an organic modifier, usually a short-chain alcohol or acetonitrile. These modifiers increase the elution strength and often improve the shape of the chromatographic peaks. The modifiers solvate the bonded stationary phase and reduce the amount of surfactant adsorbed, the effect being larger with increasing concentration and hydrophobicity of the alcohol. Selection of the $\mathrm{pH}$ in the mobile phase is also often extremely important for the resolution of complex mixtures, owing to the side acid-base reactions of many solutes. Other variables to be considered are temperature and ionic strength [1].

The chromatographer is concerned with the achievement of the optimum mobile phase that permits the separation of the composition in a mixture, in the minimum time. This task may be really difficult when two or more variables are involved in the optimisation process. The optimisation strategy may be sequential or interpretative. In a sequential strategy, the retention of the solutes is not known a priori, and each set of mobile phases is designed by taking into account the retention observed with previous eluents. In contrast, in an interpretative strategy, the experiments are designed before the optimisation process and used to fit a model that will permit the prediction of the retention of each solute. This strategy may be much more efficient and reliable. A sequential strategy will be inadequate when several local (or secondary) maxima exist (as occurs in chromatography) and may not give the best maximum, that is, the optimum.

The necessity for an adequateexperimental design becomes especially important when dealing with forms of liquid chromatography suitable for the simultaneous analysis of ionic and nonionic compounds, such as MLC, where several variables should be controlled (e.g., type and concentration of surfactant and organic modifier, $\mathrm{pH}$, temperature, and ionic strength). The method development strategy must provide the chromatographer with an answer to which variables should be used, and how to set up initial experiments to search the appropriate variable space in an effective way. 
The separation process in a micellar chromatographic system requires a structured approach in the development of practical applications. Ideally, the resolution of complex mixtures should be made and optimized in a short time, with minimal consumption of reagents.

\section{Empirical Models}

Retention in a hybrid micellar mobile system can be modelled using a procedure that utilizes the retention data of only five mobile phases [2]: four measurements at the corners of the selected two-dimensional variable space, defined by the concentrations of surfactant and modifier, and the fifth in the centre. The chromatographic data obtained (retention factor, efficiency, and asymmetry factor) are used to fit some equations. The models used for the description of retention behaviour are summarized as in Section 2.1.

\subsection{Empirical and Mechanistic Models Used in MLC}

Empirical Models. $k$ is the retention factor for a given mobile phase composition; $[M]$ is the concentration of surfactant forming micelles (total concentration of surfactant minus the critical micellar concentration, $\mathrm{CMC}) ; \varphi$ is the volume fraction of the organic solvent; $c_{0}, c_{1}, c_{2}, c_{3}$, and $c_{11}$ are fitting coefficients:

$$
\begin{gathered}
\log k=c_{0}+c_{1}[M]+c_{2} \varphi, \\
\frac{1}{k}=c_{0}+c_{1}[M], \\
\frac{1}{k}=c_{0}+c_{1}[M]+c_{2} \varphi, \\
\frac{1}{k}=c_{0}+c_{1}[M]+c_{2} \varphi+c_{3}[M] \varphi, \\
\frac{1}{k}=c_{0}+c_{1}[M]+c_{2} \varphi+c_{3}[M] \varphi+c_{11} \varphi^{2} .
\end{gathered}
$$

Mechanistic Models. $K_{\mathrm{AS}}$ is the product of the solute- (A) stationary $(\mathrm{S})$ partition coefficient by the phase ratio, and $K_{\mathrm{AM}}$ the solute-micelle $(\mathrm{M})$ association constant; $K_{\mathrm{AD}}$ and $K_{\mathrm{MD}}$ measure the relative variation produced in the concentration of solute in bulk water and micelle, respectively, in presence of modifier, taking the pure micellar solution as a reference; $K_{\mathrm{AD} 2}$ corresponds to a quadratic hyperbolic variation in $K_{\mathrm{AS}}$ and $K_{\mathrm{AM}}$ with $\varphi$ :

$$
\begin{aligned}
& k=\frac{K_{\mathrm{AS}}}{1+K_{\mathrm{AM}}[M]}, \\
& k=\frac{K_{\mathrm{AS}}\left(1 /\left(1+K_{\mathrm{AD}} \varphi\right)\right)}{1+K_{\mathrm{AM}}\left(\left(1+K_{\mathrm{MD}} \varphi\right) /\left(1+K_{\mathrm{AD}} \varphi\right)\right)[M]}, \\
& k=\frac{K_{\mathrm{AS}}\left(1 /\left(1+K_{\mathrm{AD} 1} \varphi+K_{\mathrm{AD} 2} \varphi^{2}\right)\right)}{1+K_{\mathrm{AM}}\left(\left(1+K_{\mathrm{MD}} \varphi\right) /\left(1+K_{\mathrm{AD} 1} \varphi+K_{\mathrm{AD} 2} \varphi^{2}\right)\right)[M]},
\end{aligned}
$$

$$
k=\frac{K_{\mathrm{AS}}\left(\left(1+K_{\mathrm{SD}} \varphi\right) /\left(1+K_{\mathrm{AD}} \varphi\right)\right)}{1+K_{\mathrm{AM}}\left(\left(1+K_{\mathrm{MD}} \varphi\right) /\left(1+K_{\mathrm{AD}} \varphi\right)\right)[M]},
$$

$k$

$$
=\frac{K_{\mathrm{AS}}\left(1 /\left(1+K_{\mathrm{AD} 1} \varphi+K_{\mathrm{AD} 2} \varphi^{2}\right)\right)}{1+K_{\mathrm{AM}}\left(\left(1+K_{\mathrm{MD} 1} \varphi+K_{\mathrm{MD} 2} \varphi^{2}\right) /\left(1+K_{\mathrm{AD} 1} \varphi+K_{\mathrm{AD} 2} \varphi^{2}\right)\right)[M]} .
$$

Equations (1)-(5) correspond to empirical models. In (1), it is assumed that solute retention is linearly related to the mobile phase variables within a selected portion of the space and fitted to a separate logarithmic linear function. However, the predictions obtained with (1) are not accurate enough. Retention in micellar mobile phases with a fixed amount or without organic solvent [3] has been extensively proved to be described by the hyperbolic relationship shown in (2). To model the retention of a solute at varying concentrations of both surfactant and modifier [4], (3) can be used and has yielded acceptable results for small ranges of concentrations of surfactant and modifier, but an interaction between both factors, $[M]$ and $\varphi$, exists for larger domains.

The capability of a series of empirical equations to describe the retention behaviour for any surfactant and organic solvent content has been studied [5-7]. Several models were considered where $\log k$ or $1 / k$ values were related to the micellar concentration and volume fraction of organic modifier. Logarithmic models usually yielded poorer results. Equation (4) is the simplest equation giving good predictions for both polar and moderately polar compounds, such as amino acids, sulfonamides, $\beta$-blockers, and diuretics. Equation (4) yields linear plots of $1 / k$ versus $\varphi$ at fixed concentrations of surfactant. However, for highly hydrophobic compounds, such as steroids or polycyclic aromatic hydrocarbons, the plots are nonlinear. An additional term is required to achieve more accurate descriptions, as shown in (5). The parameters in (4) and (5) should be obtained by fitting the data in experimental designs with at least four and five mobile phases, respectively. However, at least one additional measurement should be taken to check the accuracy of the fittings.

\section{Mechanistic Models}

The parameters of the empirical models of MLC are related to physicochemical constants that describe the interactions of the solutes with the three environments involved in micellar mobile phases: bulk water, micelles, and the stationary phase. A better understanding of the retention mechanism in micellar systems is provided by these models.

The mechanistic models are based on (2), which is the classical equation proposed for micellar mobile phases at a fixed volume fraction of organic modifier [2]. Equation (2) can be written as (6), which relates the retention of a solute to the concentration of monomers of surfactant in the form of micelles.

For hybrid micellar mobile phases, (6) can be expressed as (7), while (5) gives rise to (8) [8], which may suggest an 
excessive dependence of the retention on the organic solvent concentration and produce high errors when an extrapolation is made in a region of high concentration of modifier.

As a result, (9) was proposed as an alternative model for highly hydrophobic solutes, and it takes into account the additional change in the concentration of solute associated with the stationary phase produced by the presence of modifier [7]. In (9), the constants $K_{\mathrm{MD}}$ and $K_{\mathrm{AD}}$ account for the displacement of the water-micelle equilibrium, whereas $K_{\mathrm{SD}}$ and $K_{\mathrm{AD}}$ describe the modification of the water-stationary phase equilibrium. These changes are caused by the decrease in the polarity of water and the modification of the interactions of solute with micelles and stationary phase, when a modifier is added. Equation (9) provides an accurate description of the retention of solutes of a wide range of polarities, when they are eluted with hybrid mobile phases of SDS and alcohol (propanol, butanol or pentanol) [8]. For acetonitrile and tetrahydrofuran $[9,10],(10)$ fits better.

\section{Peak Shape Modelling}

The major drawback of applying MLC to practical separations is still the low chromatographic efficiency, which is caused by resistance to mass transfer in the processes involving micelles and a surfactant-modified stationary phase. This is especially important since the increase in micelle concentration causes a decrease in plate number, resulting in a varying efficiency over the variable space. Thus, it is very important to include the expected peak shape in the expression of the chromatographic quality. The complexity of the chromatographic process does not allow the use of simple equations to describe peak profiles. The best peak-profile predictions are achieved using a Gaussian equation where the standard deviation depends polynomially on the distance to the peak time (polynomial modified Gaussian model) [11]:

$$
h(t)=H_{0} e^{-0.5\left(\left(t-t_{R}\right) /\left(s_{0}+s_{1}\left(t-t_{R}\right)+s_{2}\left(t-t_{R}\right)^{2}+\cdots\right)\right)^{2},}
$$

where $h(t)$ is the predicted signal at time $t, H_{0}$ the maximal peak height, $t_{R}$ the retention time, and the coefficients $s_{i}$ are related to the width and asymmetry of the chromatographic peak. For a given solute and mobile phase, $t_{R}$ and $s_{i}$ are ideally invariable, whereas $H_{0}$ depends on the concentration. Better descriptions of peak profiles are achieved by increasing the degree of the polynomial. The use of a larger number of coefficients improves the fittings but decreases the practical application of the model. A linear standard deviation in (11) approximates real peak profiles satisfactorily. The linear equation is also useful for simulating chromatograms.

\section{Strategies to Measure the Peak Resolution}

The simulation of chromatograms requires predictions of peak retention and peak profile that are as accurate as possible. Computer optimization attempts to mimic the methodology followed by an experienced chromatographer so as to reduce the time and the effort required. A software application was developed to assist the chromatographer in the selection of the optimal composition of the mobile phase in MLC [12]. This program allows chromatograms to be simulated quickly and therefore enables the changes in the chromatograms to be observed as the user simulates variations in the composition of the mobile phase. The resolution surfaces and ease of simulation can be applied in a straightforward manner to select the best composition of the mobile phase.

Different measurements of diverse complexity have been proposed to depict chromatographic performance. Optimization criteria based on calculation of an individual or elementary resolution measurement, $r_{i}$, for the least resolved peak or peak pair is a very widely used procedure in chromatographic practice, because of its simplicity:

$$
R=\operatorname{MIN}\left(r_{i}\right) \quad 1 \leq i \leq p,
$$

where $p$ is the number of peaks or peak pairs, and $R$ is the global resolution.

This criterion is reasonable, but it considers the resolution of only one peak or peak pair, and is insensitive to the remaining peaks. In many cases, a practically identical resolution of the worst peak can be obtained, while the resolution of the other peaks can be improved. The product of peak resolutions solves this drawback, since it optimizes the resolution of all peaks in the chromatogram.

The normalized-by-mean product is conventionally applied. Section 5.1 shows (13), the global resolution functions, and elementary resolution criteria. This treatment normalizes the resolution approximately, using the mean $r_{i}$ of all the peaks in the chromatogram instead of the extreme values.

The unnormalized product of (14) seems to be a better alternative, although it can be used only with intrinsically normalized resolution measurements. This product varies from 0 (complete overlapping between at least two peaks) to 1 (full resolution of every peak in the chromatogram).

After the selection of the global resolution function, the appropriate elementary resolution criterion (Section 5.1) should be decided. Some criteria have been based on conventional elementary measurements, such as the modified selectivity in (15), peak-to-valley measurements in (16), and overlapping fraction measurements in (17).

In (16), the valley between two consecutive peaks can be measured at the time giving the largest possible distance, measured orthogonally. If the valley is observed orthogonally, this point is obvious even when there is substantial overlap.

The criterion of overlapping fractions takes into account not only positions but also peak profiles; it isolates the contribution of each component in a mixture, associating a value to each individual peak, which is not affected by the identity of its neighboring peaks, and the intrinsic normalisation facilitates understanding of the information obtained in the optimization process. 


\subsection{Global Resolution Functions and Elemental Resolution Criteria Used in MLC}

Resolution Function. $k_{i}$ and $k_{i+1}\left(k_{i+1}>k_{i}\right)$ are the retention factors of two neighboring peaks, and $\alpha_{i ; i+1}$ is the selectivity; $h_{1}$ represents the height of the signal at a specific time depicting the valley location, and $h_{2}$ is an interpolated height, measured at that time, from baseline to the line obtained by joining the maximums of the two neighboring peaks; $o_{i}^{\prime}$ is the area under a given peak overlapped by the chromatogram yielded by the remaining peaks, and $o_{i}$ the total area of the peak:

$$
\begin{gathered}
R=\frac{\prod_{i=1}^{p} r_{i}}{\left[\sum_{i=1}^{p} r_{i} / p\right]^{p}}, \\
R=\prod_{i=1}^{p} r_{i}, \\
r_{i, i+1}=1-\frac{k_{i}}{k_{i+1}}, \\
r_{i, i+1}=1-\frac{h_{1}}{h_{2}}, \\
r_{i}=1-\frac{o_{i}^{\prime}}{o_{i}} .
\end{gathered}
$$

\section{Conclusions}

The development of a micellar analytical procedure for the determination of solutes requires optimization of the column type, the type and concentration of the surfactant and organic modifier, $\mathrm{pH}$, flow rate, and temperature. In addition, the optimization strategy can be sequential or interpretive. In a sequential strategy, a set of mobile phases is designed by taking into account the retention observed with previous eluents (the retention of the solutes is not known a priori). When the experiments are designed before the optimization process and used to fit a model that will allow the retention of each solute to be predicted, the strategy is called interpretative, and, of the two, it is more efficient and reliable. The need for an adequate experimental design becomes especially important when dealing with forms of liquid chromatography suitable for the simultaneous analysis of ionic and nonionic compounds, such as MLC, in which several factors should be controlled. The strategy used to develop the method must provide answers to questions concerning the variables to be used as well as the set-up of the initial experiments that will yield an effective search of the appropriate variable space. Retention in a hybrid micellar mobile system can be modeled using a procedure that utilizes the retention data of only five mobile phases: four measurements at the corners of the selected two-dimensional variable space, defined by the concentrations of surfactant and modifier, and the fifth in the center. The chromatographic data obtained (retention factor, efficiency, and asymmetry factor) are used to fit some equations. Thus, computer optimization permits to mimic the methodology followed by an experienced chromatographer, reducing the time and the effort required.

\section{Acknowledgment}

The author wishes to thank the MEC for the FPU grant.

\section{References}

[1] M. J. Ruiz-Ángel, S. Carda-Broch, J. R. Torres-Lapasió, and M. C. García-Álvarez-Coque, "Retention mechanisms in micellar liquid chromatography," Journal of Chromatography A, vol. 1216, no. 10, pp. 1798-1814, 2009.

[2] E. D. Breyer, J. K. Strasters, A. H. Rodgers, and M. G. Khaledi, "Simultaneous optimization of variables influencing selectivity and elution strength in micellar liquid chromatography. Effect of organic modifier and micelle concentration," Journal of Chromatography, vol. 511, pp. 17-33, 1990.

[3] M. Arunyanart and L. J. Cline Love, "Model for micellar effects on liquid chromatography capacity factors and for determination of micelle-solute equilibrium constants," Analytical Chemistry, vol. 56, no. 9, pp. 1557-1561, 1984.

[4] J. R. Torres-Lapasió, M. J. Medina-Hernández, R. M. Villanueva-Camanas, and M. C. Garcia-Álvarez-Coque, "Description of the retention behaviour of solutes in micellar liquid chromatography with organic modifiers: comparison of two methods," Chromatographia, vol. 40, no. 5-6, pp. 279-286, 1995.

[5] J. R. Torres-Lapasió, R. M. Villanueva-Camañas, J. M. SanchisMallols, M. J. Medina-Hernandez, and M. C. Garcia-ÁlvarezCoque, "Modelling of the retention behaviour of solutes in micellar liquid chromatography with organic modifiers," Journal of Chromatography, vol. 639, no. 2, pp. 87-96, 1993.

[6] J. R. Torres-Lapasió, R. M. Villanueva-Camanas, J. M. SanchisMallols, M. J. Medina-Hernandez, and M. C. Garcia-ÁlvarezCoque, "Interpretive strategy for optimization of surfactant and alcohol concentration in micellar liquid chromatography," Journal of Chromatography A, vol. 677, no. 2, pp. 239-253, 1994.

[7] S. Torres-Cartas, R. M. Villanueva-Camañas, and M. C. García-Álvarez-Coque, "Modelling and optimization of the separation of steroids eluted with a micellar mobile phase of sodium dodecyl sulphate containing acetonitrile," Analytica Chimica Acta, vol. 333, no. 1-2, pp. 31-40, 1996.

[8] M. C. García-Álvarez-Coque, J. R. Torres-Lapasió, and J. J. Baeza-Baeza, "Description of the partitioning behaviour of solutes and data treatment in micellar liquid chromatography with modifiers," Analytica Chimica Acta, vol. 324, no. 2-3, pp. 163-173, 1996.

[9] S. López Grío, J. J. Baeza Baeza, and M. C. García ÁlvarezCoque, "Modelling of the elution behaviour in hybrid micellar eluents with different organic modifiers," Analytica Chimica Acta, vol. 381, no. 2-3, pp. 275-285, 1999.

[10] S. J. López-Grío, G. Vivó-Truyols, J. R. Torres-Lapasió, and M. C. García-Álvarez-Coque, "Resolution assessment and performance of several organic modifiers in hybrid micellar liquid chromatography," Analytica Chimica Acta, vol. 433, no. 2, pp. 187-198, 2001.

[11] J. R. Torres-Lapasió, J. J. Baeza-Baeza, and M. C. GarcíaÁlvarez-Coque, "A Model for the Description, Simulation, and 
Deconvolution of Skewed Chromatographic Peaks," Analytical Chemistry, vol. 69, no. 18, pp. 3822-3831, 1997.

[12] J. R. Torres-Lapasió, Michrom Software, Marcel-Dekker, New York, NY, USA, 2000. 


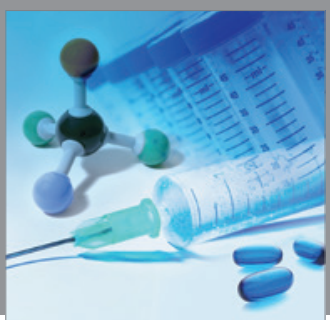

International Journal of

Medicinal Chemistry

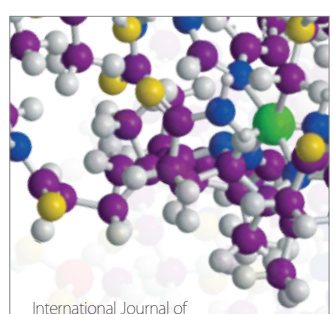

Carbohydrate Chemistry

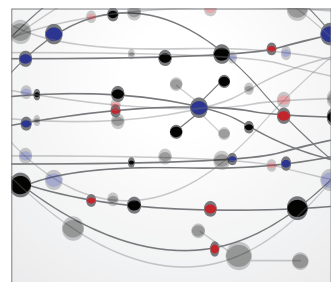

The Scientific World Journal
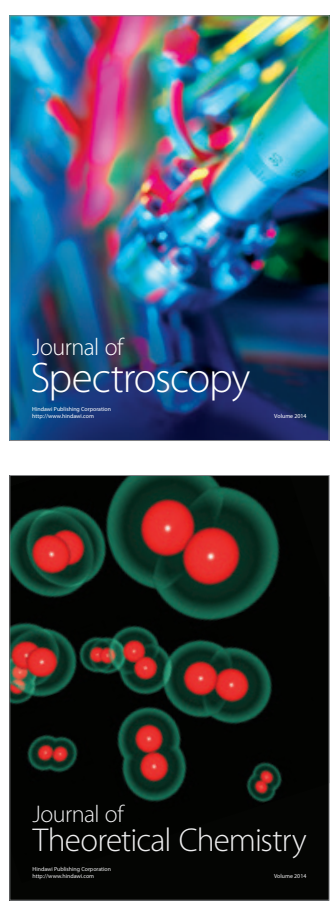
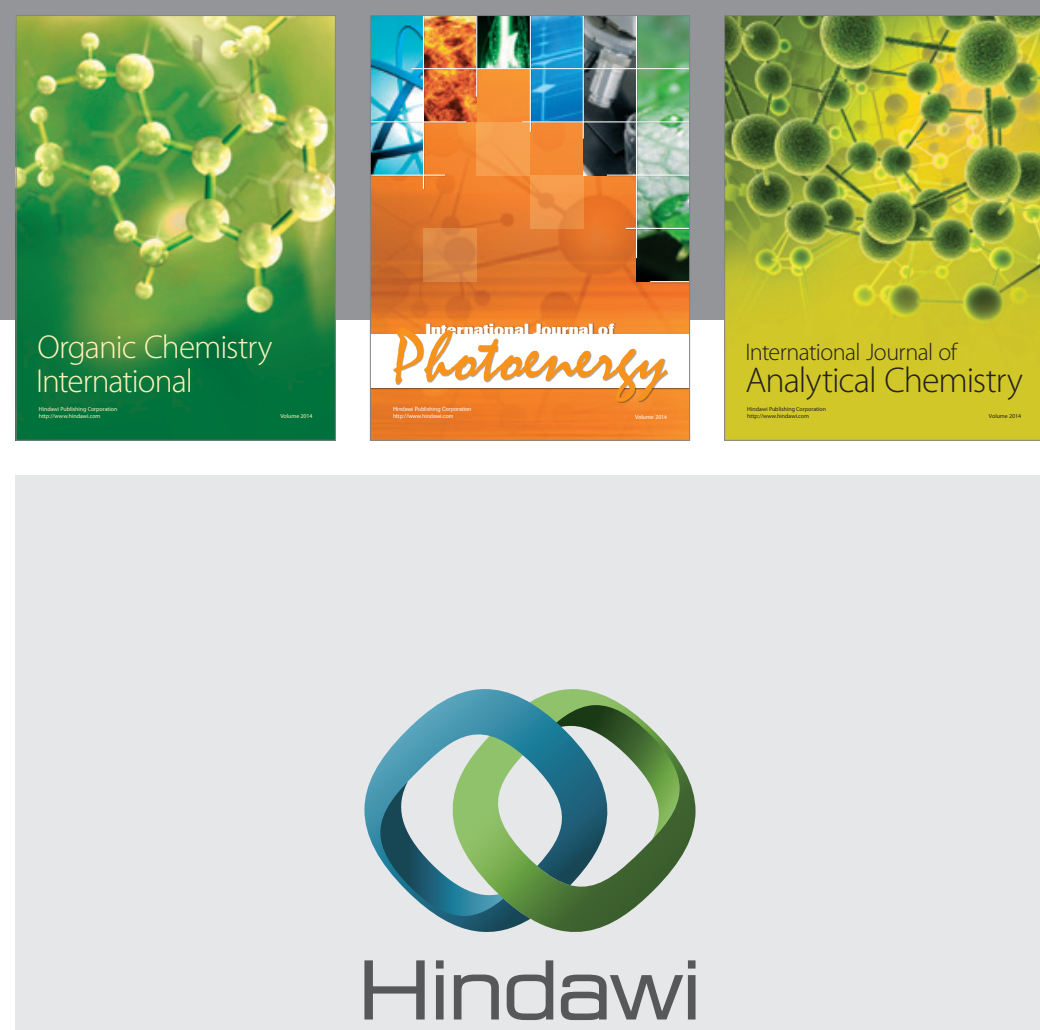

Submit your manuscripts at

http://www.hindawi.com
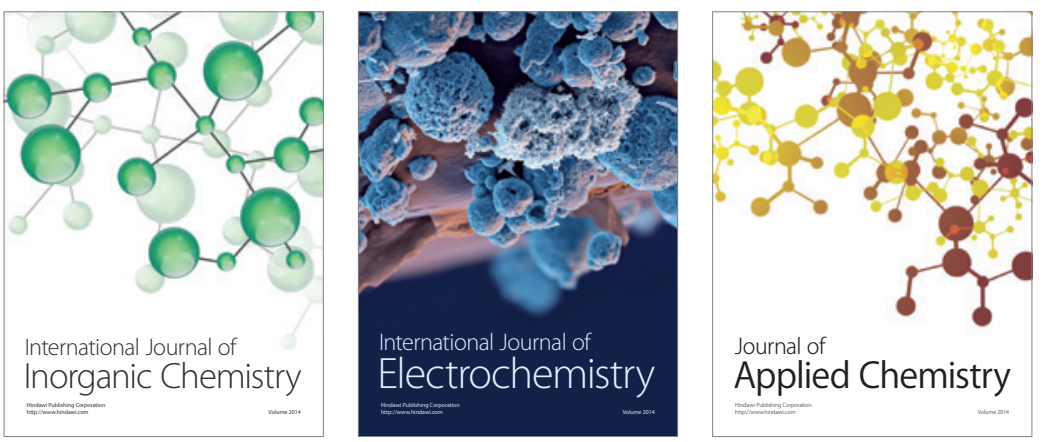

Journal of

Applied Chemistry
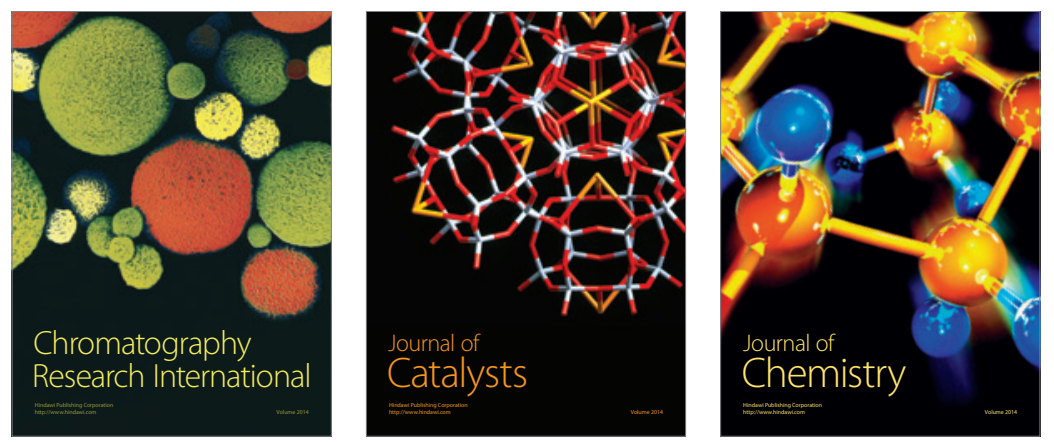
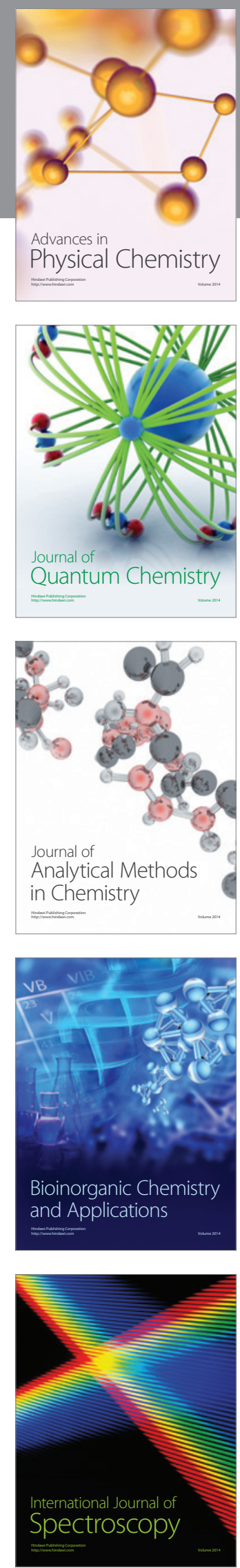